\title{
¿POR QUE HA «TRIUNFADO» EL JAPON? A PROPOSITO DE LA OBRA DE M. MORISHIMA
}

\author{
RAFAEL DOBADO GONZALEZ \\ Universidad Complutense
}

La aparición en España de la última obra de Michio Morishima ' constituye una agradable sorpresa. Varios son los motivos que obligan a saludar la entrega al lector de lengua castellana de Por qué ba "triunfado» el Japón.

En primer lugar, su contenido desborda el marco temático que algunas interpretaciones, tal vez excesivamente restrictivas, asignan a la disciplina económica. A lo largo de sus páginas se va construyendo un discurso que incorpora elementos diversos procedentes de distintos campos de las ciencias sociales. Las creencias religiosas, el pensamiento jurídico y político, los valores ideológicos, el protagonismo del Estado y de las grandes empresas en la economía, la incorporación de la ciencia y de la tecnología extranjeras, el ejército y el expansionismo militar, la dualidad de la estructura económica, la educación y el mercado de trabajo serán repetidamente empleados en la tarea de explicar y matizar el «triunfo" de Japón en la persecución del desarrollo económico capitalista. Uno de los méritos de la obra estriba en abordar con perspectiva amplia y talante nada dogmático el complejo proceso de transformaciones experimentado por la sociedad japonesa desde mediados del siglo XIX, momento en el que a la forzada ruptura de un casi total aislamiento exterior que duraba doscientos cincuenta años se une la instauración de un Estado que liquida la organización política feudal y sienta las bases para el funcionamiento de una economía capitalista. Posteriormente, la sociedad japonesa abordó la industrialización con mejores resultados que ninguna otra no occidental. Para Morishima, paradójicamente, la adopción de las técnicas industriales foráneas se vio favorecida por un ethos nacional muy distinto al de los países occidentales, el cual, por otra parte, dotó al capitalismo japonés de ciertas diferencias respecto al modelo de «libre empresa». En su rastreo de las relaciones entre la cultura material y el mundo de las ideas, Morishima se remontará hasta el siglo vi, cuando llegan a Japón el budismo, el confu-

' Morishima (1984). 
cianismo y el taoísmo, procedentes del continente asiático. Esta inmersión en el lejano pasado japonés no es gratuita, pues el principal objetivo de Morishima será dilucidar el papel desempeñado por los valores ideológicos primordiales, ámbito de la vida social apenas influido por la violenta irrupción de Occidente, en la asimilación de conocimientos técnicos provenientes del exterior y en la creación de unas formas organizativas y de una lógica de comportamiento de los agentes económicos que no reproducen exactamente las pautas británicas clásicas. Para Morishima, la intervención de las clases dominantes consiguió que las creencias religiosas antes citadas adoptaran en Japón rasgos diferenciales de amplio alcance respecto a sus versiones originarias. Una vez modificadas, constituirian, junto a la educación, el principal mecanismo difusor de unos valores ideológicos que realizaron una destacada aportación al rápido y original desarrollo del capitalismo japonés. Aunque no novedoso, el planteamiento de Morishima convierte a Por qué ha "triunfado» el Japón en un sano ejercicio de libertad intelectual, en unos momentos en los que la economía muestra ciertos síntomas de agotamiento que bien pudieran deberse a una endogamia excesiva.

En segundo lugar, un motivo adicional de interés reside en que la obra que comentamos se ocupa de una sociedad que, como acertadamente señala Touraine ${ }^{2}$, sustituye en nuestros días a los EE. UU. como modelo de un éxito que no es sólo económico, sino también político y social. A comienzos de la presente década, tras experimentar, a mediados de los años setenta, una crisis de gran intensidad que frenó bruscamente un crecimiento que todavía, caso único en el mundo desarrollado, se situaba en 1972 y 1973 en tasas cercanas al 9 por 100 anual, la economía japonesa evidencia una recuperación que contrasta con el estancamiento de la mayor parte de las economías occidentales. Impulsado principalmente por las exportaciones, el crecimiento del PIB coincide con el logro de grandes superávit por cuenta corriente y el mantenimiento de unas tasas de inflación y desempleo inferiores a las de los restantes miembros de la OCDE. La mirada de muchos observadores occidentales se dirige hacia Japón a la búsqueda de soluciones a los problemas propios. Al mismo tiempo, algunas características estructurales de la economía japonesa (la elevada proporción del ahorro bruto y de la formación bruta de capital en el PIB, la alta proporción de los excedentes empresariales en la renta nacional, la baja presión fiscal y la relativa debilidad de las resistencias ofrecidas por el aparato productivo y los mecanismos distributivos a la implantación de políticas de ajuste) recibían comentarios elogiosos.

Así, Japón se nos presenta como una flamante potencia económica de brillante porvenir. Varios son los factores explicativos de esta sólida posición:

\footnotetext{
Touraine (1984).
} 
una base tecnológica de punta de reciente adquisición, una especial habilidad para adoptar y abandonar líneas de especialización en el mercado mundial acordes con las cambiantes dotaciones de factores, una capacidad exportadora a prueba de recesiones comerciales, una protección del mercado interior tanto más eficaz cuanto no se basa exclusivamente en barreras arancelarias y una colaboración empresas-administración-sindicatos que despierta la envidia de numerosos países occidentales. Además, como prueba la permanencia del Partido Liberal Democrático en el Gobierno desde hace treinta años, su estabilidad política parece indiscutible, mientras que la uniformidad social reduce la disidencia y los enfrentamientos a manifestaciones marginales de descontento, incapaces de poner en cuestión ninguno de los principios fundamentales del orden social. Este idílico marco resulta terreno abonado para el florecimiento de valores ideológicos como la disciplina, el esfuerzo, la competitividad y el ahorro, que, por unas u otras razones, habrían visto mermada su influencia en la vieja Europa y en la Norteamérica prerreaganiana.

Ahora bien, la presentación que desde numerosos sectores se hace de Japón como modelo de superación de la crisis no surge tan sólo como resultado de un examen objetivo de ciertos datos (inflación, desempleo, crecimiento, balanza comercial, desarrollo tecnológico, etc.), sino que también persigue fines prácticos político-económicos predeterminados (reducción de la presencia del Estado en aquellos ámbitos en los que la iniciativa privada podría actualmente obtener beneficios, modificación de la distribución funcional de la renta en favor de los excedentes empresariales, disminución de la progresividad fiscal, debilitamiento de la capacidad de respuesta de los sindicatos, etc.). En otras palabras, la admiración ante el comportamiento de la economía japonesa en estos últimos años forma parte del discurso ideológico de empresarios y políticos occidentales. La lectura de revistas como L'Expansion, Business Week y similares o el repaso a las declaraciones de algunos gobernantes europeos, en particular de los franceses, resultan muy ilustrativas a este respecto.

Por otra parte, es bien sabido que la producción de ideología no siempre se sustenta en un conocimiento profundo de la realidad, lo cual parece ser especialmente cierto en el caso que nos ocupa. La fiabilidad de la exposición oficial de la economía japonesa ofrece dudas motivadas por la utilización que las autoridades hacen de unos criterios estadísticos distintos de los usuales en los países occidentales. Así, las cifras oficiales se elaboran sobre bases estadísticas que sistemáticamente tienden a «adornar» la realidad, impidiendo una comparación rigurosa con las economías europea o norteamericana. Jorland ${ }^{3}$ afirma que el índice de precios al consumo dista de reflejar el coste

\footnotetext{
'Jorland (1984), pp. 178-179.
} 
real de la vida y que el dasimpleo alcanzaría un valor doble o triple si se empleasen los criterios occidentales. Según Santucci ${ }^{4}$, la inflación y el desempleo están infravalorados, mientras que con el crecimiento y el salario medio por trabajador ocurre lo contrario. De ser esto cierto, y las pruebas aportadas por los citados autores son contundentes, muchas de las opiniones emitidas acerca de la economía japonesa deberían ser revisadas. Para Santucci, la actitud gubernamental respondería a dos objetivos: la movilización interior de la población y la propaganda exterior. Este último, al menos, ha cosechado frutos $\mathrm{y}$, muy probablemente, el primero también.

En el libro de Morishima puede obtenerse una valiosa información relativa a ciertas peculiaridades de la economía japonesa que contribuyen a explicar su funcionamiento actual y que no siempre son tenidas en cuenta en los ensayos económicos de inspiración menos heterodoxa. Por tanto, Por qué ha "triunfado» el Japón colabora en la tarea de disipar la opacidad que envuelve a la realidad económica japonesa y que, extendida al conjunto de la sociedad, era señalada recientemente por Gómez de Liaño ${ }^{5}$.

Por otra parte, si para los hombres de negocios, funcionarios públicos y economistas académicos, el Japón actual ejerce una poderosa atracción, su historia económica despierta desde hace tiempo el interés de los practicantes de esta disciplina ${ }^{6}$. Dos hechos sobresalen en el pasado de la economía japonesa: la rápida transición del feudalismo al capitalismo tras la revolución Meiji y el espectacular crecimiento económico que arranca en la década de los cincuenta ${ }^{7}$. Ambos períodos de su historia económica bastarían por sí solos para convertir el caso japonés en una experiencia idónea para la contrastación de teorías del desarrollo y para la evaluación de las consecuencias sociales del cambio económico. El ensayo de Morishima, que intenta arrojar nueva luz sobre las causas que explican la extraordinaria capacidad de la economia y la sociedad japonesas para experimentar transformaciones profundas y veloces, no puede sino merecer la atención del lector atraído por estos temas.

Un tercer motivo de interés reside en la propia personalidad del autor. Bien conocido hasta ahora por sus trabajos en el campo de la teoría económica, la publicación de Por qué ba «triunfado» el Japón supone un brusco

- Santucci (1985).

' Gómez de Liaño (1984).

- Véanse Allen (1980), Aujac (1983), Bix (1982), Francks (1984), Goldsmith (1983), Halliday y McCormack (1975), Klein y Ohkawa (1968), Maddison (1971), Makoto (1983), Nakamura (1981) (1983), Tokado (1979) y Yamamoto (1982).

${ }^{7}$ Según los datos of recidos por el Historical Statistics, 1960-1983, de la OCDE, la tasa media interanual de crecimiento del PIB fue, en $1960-68$ y $1968-73$, 10,5 y 8,8 por 100 en términos reales, respectivamente. A efectos de comparación, para el conjunto de la citada organización, dichas tasas fueron 5,1 y 4,8 por 100 . 
cambio en la trayectoria intelectual de Morishima. No deja de sorprender que un teórico de la economía, que sistemáticamente recurría a las matemáticas en su labor analítica, se introduzca en un terreno tan distinto al que le era habitual como el recorrido en el ensayo que nos ocupa. Un repaso de sus anteriores obras, aunque carezca de la extensión y profundidad merecidas, facilitará el acercamiento a ésta su primera incursión en tareas estrictamente históricas.

Una de las áreas de especialización de Morishima es la teoría del crecimiento. Resultado de sus investigaciones en este campo son Equilibrium, Stability and Growth ${ }^{8}$ y Theory of Economic Growth ${ }^{9}$. Esta última pretende contribuir a la formulación de una teoría del equilibrio dinámico. Para ello analiza el crecimiento económico a partir de la teoría del equilibrio general. Su línea de razonamiento se basa en Walras y Von Neumann. Morishima, tras pasar revista al tratamiento neoclásico del equilibrio del crecimiento, a las implicaciones de la «revolución Von Neumann» y a otras aportaciones posteriores al controvertido tema del crecimiento, concluye con un modelo de optimización simultánea del capital y de la población de utilidad para los historiadores de la economía, pues introduce elementos como son las relaciones entre el salario real, el crecimiento demográfico y la productividad, o entre la distribución de la renta y el aumento del producto social.

En The Economic Theory of Modern Society ${ }^{10}$, Morishima parte del obligado reconocimiento de la existencia de esas economías no capitalistas que suelen quedar fuera del ámbito de análisis de buena parte de los estudios de teoría económica. El grado de abstracción requerido por el método teórico permite a Morishima encontrar un amplio y significativo conjunto de características comunes (población, Estado, empresas, trabajadores, mecanismos de asignación de recursos y de organización de la producción, racionalidad, viabilidad, etc.) que justificaría la utilización del término «economía moderna» en los casos de la URSS, Suecia, Yugoslavia o los EE. UU., por ejemplo. Esa «economía moderna» elegida como objeto de estudio será estática, carecerá de los sectores exterior y monetario, limitándose el horizonte temporal al corto plazo. En las tres partes de la obra se procede al examen de la producción, la distribución, el consumo, la asignación de recursos, el empleo y el sector público.

En esta obra, a pesar de su carácter eminentemente teórico, dos aspectos importantes de la economía japonesa, uno general y otro particular, reciben la atención de Morishima. Por un lado, el autor se remonta a la revolución Meiji para subrayar la influencia de los avatares históricos propios de cada

Morishima (1964).

Morishima (1969).

10 Morishima (1976). 
sociedad en la conformación de su estructura económica. Esto es, utiliza un momento histórico de especial trascendencia, la aparición del capitalismo en Japón, a fin de explicar el origen de las diferencias existentes entre realidades concretas (la moderna economía japonesa y otras economías modernas), por más que todas pertenezcan al mismo modelo teórico de sistema económico, el capitalista. Así, los rasgos monopolistas, corporativistas e intervencionistas del capitalismo japonés derivarían del protagonismo ejercido por burócratas, militares y enseñantes en la clase dirigente surgida de la revolución Meiji y de la temprana constitución de los zaibatsu, grandes conglomerados empresariales, estrechamente ligados al poder político, tras la venta de los activos reales públicos motivada por la crisis financiera de 1881-84. La peculiar vía japonesa al capitalismo explica también los logros y fracasos de su proceso de modernización. Entre los primeros, cabe destacar las altas tasas de crecimiento económico; sobresalen, entre los segundos, la desigual distribución de la renta entre los sectores moderno y atrasado que componen el dualismo de la estructura económica japonesa y el desempleo encubierto existente en el segundo de los sectores citados. Por otro lado, este último aspecto será tratado detenida e independientemente en el capítulo final de la obra. Para Morishima, la permanencia de altos niveles de desempleo encubierto en un sector atrasado de magnitudes significativas es un resultado directo de la estructura dual de la economía japonesa. Esta, a su vez, surge como consecuencia de un modelo de modernización basado en el rápido crecimiento del sector avanzado y en el mantenimiento del atrasado como reserva de fuerza de trabajo, funciones ambas cuya asignación responde a decisiones conscientemente adoptadas por la clase dirigente del proceso de modernización desequilibrada de la economía japonesa.

En Por qué ba "triunfado» el Japón reaparecerán, si bien expuestas con mayor detalle y en interrelación con los valores ideológicos difundidos precisamente por ese sector dirigente en razón de su coherencia con el modelo de industrialización implantado, las líneas maestras de esta interpretación del proceso modernizador de la economía japonesa que Morishima expone en The Economic Theory of Modern Society.

Es, probablemente, el análisis de la obra de Marx desde la óptica de la economía matemática la faceta mejor conocida de la labor de Morishima, constituyendo una referencia casi obligada en las polémicas actuales de cierta altura en torno a la teoría económica marxista.

En un artículo, elaborado junto a Seton ", conseguía algo que el propio Marx no logró. Se trata de la demostración formalmente rigurosa del famoso Teorema Fundamental Marxista, el cual sostiene que la tasa de beneficios es

"Morishima y Seton (1961). 
positiva si, y sólo si, la tasa de explotación, definida como el cociente entre el trabajo excedente y el trabajo necesario, es positiva.

En La teoría económica de Marx ${ }^{12}$ se pasa revista a los puntos básicos de la contribución de Marx al análisis económico: la teoría del valor trabajo, la teoría de la explotación, el problema de la transformación de los valores en precios, los esquemas de reproducción simple y ampliada, la ley de la población, las tendencias a largo plazo del sistema y la rotación del capital. Con esta obra, Morishima pretende tender un puente entre el pensamiento marxista y el neoclásico o, más exactamente, presentar una teoría Marx-Von Neumann que significaría el "nacimiento de una nueva etapa del desarrollo de nuestra ciencia” ${ }^{13}$. Para ello, no duda en reivindicar la figura de Marx como «economista puramente académico», calificativo un tanto equívoco, pero que no deja de expresar el respeto por su labor en el campo de la teoría económica $y$, por sorprendente que pueda parecer a algunos, por sus aportaciones pioneras a la economía matemática. En esa misma línea de reconocimiento de un papel no siempre admitido se sitúa su afirmación de que corresponde a Marx el mérito de ser el «fundador de la teoría del equilibrio dinámico general, núcleo fundamental de la teoría económica» ${ }^{14}$. Sin embargo, Morishima tampoco ahorra críticas a la teoría marxista. A su juicio, la necesaria consideración de fenómenos como la producción conjunta, la elección de técnicas y el trabajo heterogéneo obliga al abandono de la teoría del valor trabajo. Hecho éste que, por otra parte, no tendría repercusiones especialmente graves, pues la teoría marxista sería tan concebible sin la teoría del valor trabajo como la walrasiana sin la teoría de la utilidad. Para Morishima, La teoría económica de Marx constituye una parte más de su investigación sobre el crecimiento económico.

En otro artículo, titulado Marx in the Ligth of Modern Theory ${ }^{15}$, presenta una versión generalizada del Teorema Fundamental Marxista según la cual una tasa de explotación positiva es condición necesaria y suficiente para la existencia de una tasa de beneficio y de una capacidad de crecimiento positivas.

Resulta indiscutible que Morishima ha contribuido positivamente a la recuperación del pensamiento de Marx, así como a poner de manifiesto algunas de las debilidades que aquejan a la consistencia lógica de ciertas piezas básicas de su esquema económico. No obstante, considero que Morishima no parece tener suficientemente en cuenta un aspecto crucial a la hora de juzgar la teoría del valor trabajo. Se trata de la función que ésta cumple dentro de la construcción analítica marxista. En efecto, hoy día se admite ampliamente

\footnotetext{
${ }^{12}$ Morishima (1977).

13 Ibidem, p. 18.

14 Ibidem, p. 17.

15 Morishima (1974).
} 
que la solución ofrecida por Marx para obtener precios de producción a partir de valores (el tan debatido problema de la transformación) es errónea o, más bien, simplemente innecesaria, pero también se acepta generalmente que este hecho no se le escapaba y que, por otra parte, no le concedía mayor importancia a esta insuficiencia formal. La causa de esta actitud obedece a que el objetivo de la teoría del valor no era servir de paso previo a la elaboración de una teoría acabada de los precios, sino otro de muy distinta índole. Preferentemente, su propósito consistía en hacer evidentes las diferencias existentes entre la esfera de la producción y la de la circulación y subrayar que la primera es la determinante en el modo de producción capitalista, pues es allí donde se produce la extracción de trabajo a partir de la fuerza de trabajo y la consiguiente aparición de plusvalía en forma de trabajo apropiado y no remunerado.

Posteriormente, Morishima ha publicado nuevos escritos que insisten en los temas que habitualmente han venido ocupando su atención ${ }^{16}$. Como puede apreciarse en este precipitado repaso, es la suya una aportación extensa y rica a la teoría económica contemporánea, en la que, junto al buen conocimiento de las diferentes escuelas y al intenso empleo de las matemáticas, se detecta una actitud ecléctica que le permite incorporar elementos de diversa procedencia a su meritoria labor analítica.

A continuación entraremos de lleno en el comentario de Por qué ba «triunfado» el Japón. La obra consta de introducción, cinco capítulos y conclusión. Desde la óptica del lector pueden distinguirse tres componentes bien diferenciados: un planteamiento general del objeto de estudio y del enfoque elegidos, poniendo el énfasis en la cuestión religiosa y en las características peculiares del capitalismo japonés; una interpretación de la transición del feudalismo al capitalismo y una descripción de algunos de los rasgos más llamativos de la economía japonesa desde la revolución Meiji hasta nuestros días; una abundante información sobre ciertos aspectos de la historia política, ideológica y económica de Japón. El primer componente ocupa la introducción, el capítulo primero y la conclusión, aunque, lógicamente, se desliza, en repetidas ocasiones de manera explícita, a lo largo de toda la obra. El segundo se expone en los restantes capítulos. El tercero está presente en las diversas partes del libro.

Tras contraponer sucintamente las opiniones de Marx y Weber en lo

${ }^{16}$ Morishima (1978) y Morishima y Catephores (1978). 
que a las relaciones entre el mundo de lo material y el de los valores éticos se refiere, Morishima, siguiendo al segundo de los pensadores citados, asigna a la peculiar religiosidad nacional y, especialmente, a su componente confuciano, un papel clave a la hora de explicar particularidades significativas del capitalismo japonés como serían el nacionalismo, el paternalismo y el antiindividualismo. Así, la reinterpretación del confucianismo chino elaborada por la clase dominante japonesa, primando el valor de la lealtad al superior jerárquico, no a la propia conciencia, y relegando el de la benevolencia, virtud básica en la formulación confuciana original, convirtió el respeto a la autoridad establecida en un principio fundamental del comportamiento individual y colectivo. Al mismo tiempo, impidió el surgimiento de normas de conducta que pudieran propiciar la aparición de sentimientos individualistas. Por otra parte, el sintoísmo japonés, que procedería también de una religión china, el taoísmo, contribuirá al fortalecimiento del elemento nacionalista de la cultura nipona, al ascenso del emperador a la condición de divinidad y al arraigo del culto de la figura imperial entre la población. Especial importancia concede Morishima a la «Reforma Taika» (645-649), proyecto político que significaría la puesta en práctica de un modelo administrativo y legislativo inspirado en el recientemente introducido confucianismo. El florecimiento cultural que siguió a la reorganización de la sociedad reflejó las influencias china y coreana, al tiempo que la clase dirigente y el Estado quedarían impregnados de los valores surgidos de la reinterpretación japonesa del confucianismo. El proceso de feudalización de la sociedad japonesa, iniciado a finales del siglo xiI y comienzos del XIIr, traería consigo un reforzamiento de la virtud confuciana de la lealtad, pero entendida en su versión japonesa. Durante el bakufu Tokugawa, que se extendió desde los primeros años del siglo xvrr hasta la revolución Meiji, la ética confuciana, inicialmente reservada a la aristocracia y los guerreros, acabaría extendiéndose a otros sectores de la sociedad (terratenientes y comerciantes). A partir de 1868, el establecimiento por el Estado moderno de la enseñanza y el servicio militar obligatorios serviría de vehículo a la difusión de los valores confucianos entre la práctica totalidad de la población. Así, en un dilatado proceso histórico, la visión confuciana del mundo constituiría uno de los pilares básicos de la ideología nacional. El resurgimiento del sintoísmo durante la primera mitad del siglo $\mathrm{xIX}$, directamente conectado con la posterior restauración del poder imperial, completa el panorama religioso que sirve de trasfondo a la industrialización japonesa y a la política expansionista iniciada poco después de la ruptura del secular aislamiento exterior y concluida, dramáticamente, con la derrota militar en 1945.

En estrecha dependencia con un código de valores que permitía a la clase dominante un amplio margen de maniobra, la economía japonesa presentará, además de los rasgos ya mencionados, una estrecha vinculación entre los gran- 
des grupos financieros e industriales, los zaibatsu, y el Estado. Al mismo tiempo, la desigual introducción de la tecnología occidental en los diferentes sectores productivos centrará la modernización en aquellos más acordes con los proyectos de la clase dirigente (minería, textil, siderurgia, construcción naval, armamento y comunicaciones) y mantendrá otros (agricultura, servicios e industrias de bienes de consumo tradicional) ajenos a los cambios en las formas productivas. El rápido cambio económico dio origen a una marcada dualidad y comportó elevados costes sociales, apareciendo al observador como un modelo de eficacia en la implantación de unas estructuras productivas y distributivas acordes con los objetivos de la clase dirigente, que alcanzaría su máxima expresión en la década de los treinta de este siglo, cuando la militarización y el intervencionismo estatal conduzcan inevitablemente al enfrentamiento bélico.

Después de 1945, la política de ocupación persiguió inicialmente una democratización profunda de la sociedad japonesa. Sin embargo, la victoria comunista en China y la guerra de Corea traerían consigo un cambio drástico en la actitud del ocupante. Consideraciones estratégicas hicieron pasar a primer plano la necesidad de contar con un aliado poderoso en el Extremo Oriente. Así, al amparo del nuevo papel, «el capitalismo japonés renació como el Ave Fénix, bajo una forma casi idéntica a la que tenía antes de la guerra» ${ }^{17}$, aunque ahora la conquista de cuotas crecientes del mercado mundial por medios pacíficos sustituiría al expansionismo militar de antaño.

Para Morishima, la asimilación de las técnicas y de las formas productivas occidentales que conduciría a la industrialización se vio favorecida por las bases sentadas al final del período Tokugawa, persiguió finalidades políticas (preservación de la soberanía nacional) y adoptó unas formas que reflejan la influencia de los valores ideológicos nacionales. Este último hecho se aprecia, en especial, a través del comportamiento de los agentes económicos. Las relaciones entre el Estado, las empresas y los trabajadores vienen determinadas, al igual que la finalidad última de sus actuaciones, por los valores sociales: el nacionalismo, el paternalismo, la supeditación de lo individual a lo colectivo y la lealtad, reduciendo las fricciones entre intereses contrapuestos, aseguran la cooperación interna necesaria para la competencia exterior.

\section{III}

Una vez expuesto, aunque sólo sea a grandes rasgos, el contenido de la obra comentada, pasaremos inmediatamente a presentar una valoración crítica

${ }^{17}$ Morishima (1984), p. 202. 
del mismo centrada en las piezas claves del discurso de Morishima y en los puntos más estrechamente conectados con la historia de la economía japonesa.

En primer lugar, a la vista del parcial análisis del «triunfo» japonés efectuado por Morishima, tal vez sea innecesario subrayar que el título de la obra no responde plenamente a lo que en ella puede encontrarse. El subtítulo, Tecnologia occidental y mentalidad japonesa, sí parece más adecuado para expresar el hilo argumental que vertebra la monografía. A mi juicio, la lectura puede resultar decepcionante si se pretende hallar un estudio en profundidad del proceso global de desarrollo del capitalismo japonés enteramente satisfactorio. Ahora bien, cuando se presta atención a la advertencia del propio Morishima de que su objetivo es menos ambicioso, limitándose a tratar "un aspecto de la cuestión", la sensación de hallarse en presencia de un intento explicativo no del todo bien resuelto desaparece. Para su autor, Por qué ba «triunfado» el Japón representa una contribución a un extenso campo de investigaciones: la comparación internacional de los sistemas económicos a partir de las respectivas religiones. No por ello se deja de ofrecer una interpretación, tal vez excesivamente sintética, de las líneas maestras del desarrollo capitalista en Japón. Se volverá sobre ella más adelante.

En segundo lugar, el método empleado por Morishima para explicar la industrialización japonesa, consistente en considerarla como resultado de factores extraeconómicos, entre los cuales la ideología y, especialmente, la religión ocupan un papel de primer orden, guarda un notable paralelismo con otros enfoques. A este respecto, no puede pasarse por alto que la influencia de Weber es, además de notoria, explícitamente reconocida por Morishima ${ }^{18}$. Pero también otros autores han abordado el examen del cambio técnico-económico en Japón desde posiciones exteriores al ámbito de la economía y de la tecnología.

Recientemente, Aujac ${ }^{19}$ ha analizado la industrialización japonesa desde una perspectiva original. Partiendo del concepto «orden social global», prácticamente equivalente al de «cultura nacional», describe las características del orden implantado tras la revolución Meiji, para concluir que dicho orden, en el que una clase dirigente dispone de un proyecto histórico, dentro del cual la industrialización desempeña funciones básicas, y de los medios financieros para impulsarlo, así como de la capacidad político-ideológica para ejercer una hegemonía incontestada sobre el conjunto de la sociedad, sería un «orden industrializante». A mi juicio, el planteamiento teórico de Aujac posee una mayor formalización y amplitud que el adoptado por Morishima.

\footnotetext{
14 «... se trata de estudiar las cuestiones sugeridas por Weber en relación con el Japón». Morishima (1984), p. 14.

19 Aujac (1983).
} 
Moritani ${ }^{20}$ también se ha sentido atraído por investigar en un campo y desde una óptica muy próximos a los que aquí nos ocupan. Se trata, en este caso, de dilucidar las relaciones existentes entre el carácter nacional y la asimilación de la tecnología occidental, primer paso hacia la consecución de una base tecnológica propia, en tres países, China, Corea y Japón, que coinciden al menos en un elemento destacado de la vida social como es la difusión desde antiguo en todos ellos del confucianismo. Sin embargo, Moritani sostiene que fue precisamente la menor presencia del confucianismo en la sociedad japonesa, donde no pasaría de ser el código de conducta de los señores feudales y de sus dependientes más directos, un factor diferencial que contribuye significativamente a explicar una mucho más temprana y exitosa industrialización. Así, una vía de aproximación idéntica a un mismo objeto de estudio conduce a una conclusión opuesta.

Sin duda, más sorprendente resulta la semejanza entre la tesis mantenida a lo largo de las páginas de Por qué ba «triunfado» el Japón y la que, a comienzos de siglo, presentaba un español, Sales y Ferré, en su libro titulado La transformación del Japón ${ }^{21}$. Si descontamos las naturales diferencias que por fuerza separan la labor de tratadistas de las ciencias sociales de tiempos, lugares $\mathrm{y}$, presumiblemente, personalidades tan distintas entre sí, el parecido dista de ser superficial. Dos son las causas de las transformaciones económicas y políticas iniciadas en Japón en la segunda mitad del siglo xIx. Por un lado, la previa revitalización del sintoísmo propició la recuperación por el emperador del poder político efectivo. Por otro lado, la rápida asimilación de ciertos elementos de la cultura europea, en especial de la tecnología y de las formas productivas, fue impulsada por el carácter esencialmente militar del Estado. Ahora bien, tanto una como otra habrían sido facilitadas por valores sociales como el «sentimiento guerrero», la obediencia y el hábito de sacrificio, en particular por estos dos últimos. Como puede apreciarse, la religión recibe también por parte de Sales y Ferré un tratamiento preferente como elemento de su modelo explicativo de la modernización japonesa. Llamativo ejemplo de afinidad entre los planteamientos de ambos autores, los dos detectan en la sociedad japonesa un "vacío emocional» originado por la forzada incorporación de nuevos valores culturales procedentes del exterior cuando todavía los tradicionales permanecen firmemente arraigados.

Resumiendo, la obra de Morishima se inserta en lo que parece constituir una corriente de pensamiento que localiza en ciertos elementos superestructurales de la sociedad japonesa (la cultura, la religión o las normas éticas) un potencial notable para la comprensión del rumbo seguido por su economía tras la revolución Meiji.

${ }^{20}$ Moritani (1981).

21 Sales y Ferré (1909). 
En tercer lugar, las características específicas atribuidas por Morishima al capitalismo japonés están definidas de manera un tanto vaga. Este hecho afecta negativamente a la consistencia de la argumentación, una de cuyas piezas claves consiste en postular la existencia de significativas diferencias entre los modelos de funcionamiento de las economías británica y japonesa. Contando exclusivamente con la información suministrada, no resulta fácil para el lector comparar de manera rigurosa ambas experiencias de desarrollo capitalista. Consecuentemente, tampoco carece de dificultades verificar si las divergencias son tan relevantes como parece sostener Morishima y si, en caso afirmativo, obedecen a la causa por él señalada. Incluso aceptando la existencia de rasgos diferenciales de cierta entidad, cabe legítimamente preguntarse por su posible relación con factores que Morishima no cita o a los que no atribuye mayor importancia.

La simple constatación de que, en el momento en que se produce la forzada integración de Japón en el mercado mundial y en la política internacional, las «reglas del juego» eran muy distintas de las imperantes cuando Gran Bretaña se industrializa y se convierte en potencia dominante o de las que han condicionado las posibilidades de desarrollo de muchas sociedades no occidentales resulta de indudable utilidad. No se trata de despreciar la incidencia de factores internos, sino solamente de recordar que, a medida que se consolida la internacionalización de las relaciones políticas y económicas, los factores externos revisten una creciente importancia. Así, ciertas circunstancias no pueden ser olvidadas sin que el análisis se resienta. Durante buena parte del proceso de industrialización japonés, la tecnología encontraba pocos obstáculos a su transferencia entre países y, consecuentemente, los costes de su incorporación al aparato productivo eran menores de lo que han sido posteriormente. Si una determinada mentalidad colectiva puede favorecer la adopción de técnicas foráneas, hechos como el citado tampoco dejan de hacerlo. Por otra parte, a la hora de valorar el éxito japonés en este terreno, debe tenerse en cuenta que todavía hoy, pese al espectacular avance experimentado en ciertos sectores, Japón es un importador neto de tecnología.

La comparación internacional permite delimitar de una manera más precisa la especificidad del caso japonés. Desde este punto de vista, el expansionismo, al igual que la concentración de la actividad industrial en un reducido número de empresas de grandes dimensiones, aparece antes como una necesidad objetiva del capitalismo maduro que como un resultado de la ideología nacional. Además, dada la posición geográfica de Japón, sus victorias militares contra China y Rusia y sus logros industriales son inseparables de la debilidad de sus vecinos. Insistiendo en esta línea de razonamiento, si la preservación de la soberanía nacional se considera como un elemento favorable de cara a la consecución del objetivo industrializador sobre el que reposa el proyecto 
histórico de la clase dirigente japonesa a partir de 1868, no puede descartarse a la ligera la positiva contribución realizada por un factor tan aleatorio como es la dotación nacional de recursos naturales. En efecto ${ }_{2}$ la ausencia en Japón de recursos minerales o agrícolas bien pudo disuadir a las potencias coloniales de la época de una ocupación que, junto a ciertas dificultades (población densa, organización política eficaz, voluntad de independencia, etc.), ofrecía pocos alicientes económicos ${ }^{22}$. Así, la autonomía política y la carencia de materias primas no deben ser del todo ajenas al hecho de que Japón no se convirtiese, en la segunda mitad del siglo xix, en una economía de exportación más entre las muchas surgidas como consecuencia de la división internacional del trabajo impulsada desde los países industrializados.

El énfasis en el papel desempeñado por la ética confuciana dificulta la necesaria consideración de otros elementos que no deberían faltar en una explicación convincente del comportamiento de la clase obrera japonesa durante las primeras etapas de la industrialización. A mi juicio, la relativamente escasa contestación de los trabajadores, que, por otra parte, no conviene exagerar, no provenía tan sólo, ni principalmente, de su adoctrinamiento en ciertos valores éticos, sino de las dificultades existentes para la consolidación del movimiento obrero japonés. La abundante oferta de trabajo, el fraccionamiento de la clase trabajadora resultante de la dualidad del mercado de trabajo y de la generalización de prácticas como la emigración temporal de campesi. nos a las factorías y su posterior retorno al medio rural de origen (especialmente importante en el sector con mayor población laboral, el textil), la escasa importancia numérica del proletariado, la represión sistemática y la ausencia de una dirección política eficaz son realidades debilitadoras de un movimiento obrero para cuya desarticulación se adoptaron pronto medidas legislativas. En 1900 se dictaba la «Ley para el mantenimiento del orden públicow.

A la luz de la experiencia histórica, la identificación del «espíritu japonés» con la pasividad frente a la dominación resulta un tanto frágil. Son numerosos los ejemplos demostrativos de que el ejercicio de las diferentes formas de dominación en las sucesivas fases de la historia de la sociedad japonesa ha sido una tarea compleja y no siempre plenamente lograda sin el recurso a la violencia institucional ${ }^{23}=$

${ }^{22}$ La expansión japonasa el continente asiático estuvo motivada, en buena medida, por la búsqueda de materies primas.

${ }^{23}$ Pruebas no faltan, tanto en el Japón feudal como en el capitalista, de la existencia de enfrentamientos sociales intensos y frecuentes. Entre 1590 y 1867 se produjeron, al menos, 2.757 conflictos campesinos de mayor o menor importancia. Especial virulencia revistieron los levantamientos rurales y urbanos entre 1824 y 1839 , cuando el descontento ante la prolongada carestía del arroz se plasmó, en 1837, en un intento insurreccional en toda regla, centrado en Osaka y duramente reprimido. Tras la revolución Meiji, el nuevo sistema impositivo y el reclutamiento forzoso motivaron numerosas revueltas campesinas (aproximadamente una media anual de treinta entre 1868 y 1874). En 1910 y 1911, la 
Valgan estas consideraciones, que, como es obvio, no agotan un tema tan complejo como es la articulación de los múltiples factores presentes en el proceso de industrialización japonés, para poner de manifiesto las limitaciones del planteamiento de Morishima. La dificultad intrínseca del objetivo elegido, determinar las relaciones de lo ideológico con lo económico, noo es ajena al resultado de su meritoria labor.

A pesar de las críticas expuestas, no querría concluir sin señalar que la lectura de Por qué ba «triunfado» el Japón es muy recomendable para todos aquellos que sientan interés por el conocimiento de la sociedad japonesa $y$, especialmente, para los historiadores de la economía que estén dispuestos a entender su objeto de estudio como resultado de factores diversos. En la obra se pueden encontrar estímulos más que suficientes para suscitar la reflexión.

La consideración de los sistemas económicos como producto de los procesos históricos, la exposición de la estructura dual de la economía japonesa resultante de la acumulación centrada en un sector, la atención prestada al mercado de trabajo, a la educación, a la tecnología y a la intervención del Estado, así como la insistencia en subrayar la correspondencia entre las opciones políticas y económicas, tanto en el plano interior como en el exterior, son elementos que enriquecen el análisis de Morishima. También el acercamiento a ciertos aspectos de la historia política e ideológica, efectuado desde dentro de la propia sociedad japonesa, merece ser situado entre sus no pocos atractivos para el lector occidental.

represión se abatiría sobre el movimiento obrero japonés, uno de cuyos líderes, Kotoku Shusui, sería ejecutado en compañía de otros anarquistas. En 1918, unos 10 millones de japoneses participaron en las «revueltas del arroz». El ejército intervino en 107 puntos del país y más de 8.000 personas fueron puestas a disposición de las autoridades judiciales. En 1923, militantes del sindicalismo revolucionario serían asesinados aprovechando la conmoción creada por el terremoto de Tokio. Después de 1945, como el propio Morishima señala, el movimiento obrero resurgiría con una vitalidad espectacular. A comienzos de los años cincuenta, la «purga de rojos» demuestra, una vez más, que el «consensow japonés no surge por generación espontánea. 


\section{BIBLIOGRAFIA}

Allen, G. C. (1980): Breve bistoria económica del Japón moderno, Tecnos, Madrid.

Aujac, H. (1983): "Culture nationale el aptitude à l'industrialisation», en Revue d'Economie Politique, 93 année, núm. 1.

Bix, H. P. (1982): "Japon's New Vulnerability», en Monthly Review, vol. 34, núm. 7.

Bouissou, J. M., y FauRe, G. (coords.) (1984): Japon. Le consensus: mitbe et realités, Economica, París.

Francks, P. (1984): Technology and Agricultural Development in Pre-war Japan, Yale University Press, New Haven y Londres.

Goldsmit H, R. W. (1983): The Financial Development of Japan, 1868-1977, Yale University Press, New Haven y Londres.

Gómez de Liaño, I. (1984): «Japón, sociedad secreta», en El Pais, 6 noviembre, p. 15.

Halliday, J., y MCCormack, G. (1975): El nuevo imperialismo japonés, Siglo XXI, Madrid.

Jorland, P. (1984): «Les ouvriers iront-ils au paradis?», en Bouissou, J. M., y Faure, G. (1984), pp. 171-228.

KLEIN, L., y OH KaWA, K. (1968): Economic Growth. The Japanese Experience since the Meiji Era, Yale University Press, Homewood.

Maddison, A. (1971): Crecimiento económico en el Japón y la URSS, Fondo de Cultura Económica, México.

Makoto, I. (1983): «The Great World Crisis and Japanes Capitalism», en Capital and Class, núm. 1.

Moris н ImA, M. (1964): Equilibrium, Stability and Growth, Oxford University Press, Londres.

- (1969): Theory of Economic Growth, Clarendon Press, Oxford. Existe traducción castellana en Tecnos.

- (1974): *Marx in the Light of Modern Theory», en Econometrica, julio.

- (1976): The Economic Theory of Modern Society, Cambridge University Press, Cambridge. Existe traducción castellana en Bosch.

- (1977): La teoría económica de Marx, Tecnos, Madrid.

- (1978): «S. Bowless and H. Gintis on the Marxian Theory of Value and Heterogeneous Labor», en Cambridge Journal of Economics, septiembre.

- (1984): Por qué ba utriunfado" el Japón, Crítica, Barcelona.

- y Catep h ores, G. (1978): Value, Explotation and Growth, MacMillan, Londres.

- y SETon, S. (1961): "Aggregation in Leontief Matrices and the Labor Theory of Valuew, en Econometrica, abril.

Moritani, M. (1981): «Japan, China and Corea: A Cross-Country Comparison of Industrial Technology», en Japanese Economic Studies, verano.

Nakamura, T. (1981): The Post-war Japanese Economy, University of Tokio, Tokio.

- (1983): Economic Growth in Pre-war Japan, Yale University Press, New Haven y Londres.

Sales y Ferré, M. (1909): La transformación del Japón, Imprenta del Asilo de Huérfanos del S. C. de Jesús, Madrid.

SANTUCCI, G. (1985): «Les problèmes soulevés par la presentation statistique de la réalité économique japonaisen, en Problémes Economiques, núm. 1943, 9 octubre.

Tokado, K. (1979): «Desarrollo tecnológico del capitalismo japonés», en Información Comercial Española, núm. 552.

Touraine, A. (1984): «Le modèle japonais», en Bouissou, J. M., y Faure, G. (1984): pp. 7-28.

Yamamoto, Y. S. (1982): «El desarrollo de la ciencia y la tecnología en Japón», en Ciencia y tecnología en el mundo, Consejo Nacional de Ciencia y Tecnología, México. 